\title{
Metodología basada en análisis de decisiones para distribuir geográficamente una fuerza de ventas
}

\section{A decision analytic methodology for determining the geographical dispersion of a salesforce}

\section{Chew-Hernández Mario Luis}

Unidad de Estudios de Posgrado e Investigación, Tecnológico de

Estudios Superiores de Coacalco

Correo:mario@tesco.edu.mx

Viveros-Rosas Leopoldo

Unidad de Estudios de Posgrado e Investigación, Tecnológico de

Estudios Superiores de Coacalco

Correo: lviverosr@hotmail.com

\author{
Velázquez-Romero Verónica \\ Unidad de Estudios de Posgrado e Investigación, Tecnológico de \\ Estudios Superiores de Coacalco \\ Correo: ing_ind_amb@hotmail.com
}

\section{Resumen}

Este trabajo aborda el problema que enfrentan las comercializadoras al decidir la forma en que deben distribuir a sus vendedores entre los clientes potenciales (empresas que podrían realizar una compra) de su región de operación. Se presenta una metodología, basada en análisis de decisiones, cuyo objetivo es incorporar formalmente a esta decisión la información disponible sobre clientes potenciales y vendedores. Luego de definir métricas para las cualidades de ambos conjuntos, se usan diagramas de influencia para calcular la venta esperada de una asignación cliente potencial-vendedor dada. Pre-calculando la venta esperada de las asignaciones, la distribución de clientes potenciales entre los vendedores que maximice la venta total se obtiene resolviendo un problema de programación lineal binario. Para considerar el costo de viaje de los vendedores, dicha distribución óptima se toma como el punto inicial de un algoritmo heurístico incremental. Después de describir la metodología, se trabaja numéricamente un caso de estudio hipotético, para luego concluir sobre el potencial práctico de la misma.

Descriptores: Análisis de decisiones, logística de ventas, incertidumbre, diagramas de influencia, probabilidad subjetiva.

\begin{abstract}
This paper addresses the decision made by retailers when establishing how its salesmen are to be distributed among the potential customers (companies that may make a purchase) inside its operation range. A methodology, based on decision analysis, aiming to formally incorporate all available information about potential customers and salesmen to the decision, is presented. First, metrics for the qualities of both sets are developed; then, influence diagrams are used to calculate the expected sale of a specific pairing of potential customer and vendor. By pre-calculating the expected sale of all such pairings, the allocation of potential customers to salesmen that maximizes the total sale is the solution of a linear binary programming problem. If the travel cost of the salesmen is to be considered, this optimal allocation can be the starting point of an incremental heuristic algorithm. After stating the methodology, its numerical application to a hypothetical case study is presented, closing with remarks on its potential practical relevance.
\end{abstract}

Keywords: Decision analysis, salesforce management, uncertainty, influence diagrams, subjective probability. 


\section{INTRODUCCIÓN}

Este trabajo aborda una situación de decisión común para las compañías comercializadoras de productos: En su región de operación, la compañía tiene identificadas empresas que podrían adquirir su producto, denominadas "clientes potenciales". Entonces, debe dividir el conjunto de clientes potenciales entre sus vendedores, procurando maximizar el volumen total vendido.

Cuando se lleva a cabo esta división se cuenta con algún conocimiento sobre los clientes potenciales (p. ej. giro, tamaño, localización, si está creciendo o expandiendo actividades) y los vendedores (p. ej. experiencia, facilidad de palabra, conocimiento técnico) que puede aprovecharse para mejorar las ventas conseguidas. En la práctica, sin embargo, no se realiza algún esfuerzo por incluir este conocimiento dentro de un modelo que permita determinar las mejores asignaciones entre vendedores y clientes potenciales. Este trabajo presenta una metodología, con una perspectiva de análisis de decisiones (Howard, 1988 y Edwards et al., 2007), que permite incorporar formalmente este conocimiento a la decisión.

En el ámbito de la compra-venta industrial, existen varias propuestas de modelos para seleccionar proveedores (Chun, 2015; Kar 2015; Falagario et al., 2012), mientras que Da Silva et al. (2002) presentan un estudio empírico de los factores de este problema, entrevistando a intermediarios y proveedores. En cuanto a la administración de la fuerza de ventas de las empresas, Johnston y Cooper (1981) desarrollan un modelo para seleccionar vendedores y Rapp (2009) analiza la decisión de subcontratar la fuerza de ventas. Por su parte, Miragliotta et al. (2009) aplicaron la teoría de sistemas dinámicos al estudio del manejo de la fuerza de ventas para una corporación multi-negocio, desarrollando un simulador para comparar políticas de ventas y formular recomendaciones sobre los incentivos y el entrenamiento de los vendedores.

Estudios sobre la distribución geográfica de los miembros de la fuerza de ventas se presentan por Cravens y LaForge (1983), quienes desarrollan un sistema de soporte para decidir la dispersión de vendedores, y por las investigaciones que abordan el problema del vendedor viajero (Travelling Salesman Problem o TSP). El TSP es un problema clásico de investigación de operaciones donde se busca la ruta que debe seguir un vendedor al visitar un conjunto de clientes, de tal manera que se minimice la distancia total viajada (Taha, 2011).

Recientemente, se ha adicionado incertidumbre al TSP, produciendo el TSP estocástico o aleatorio. Maggioni et al. (2014) introducen costos de transporte esto- cásticos y proponen usar información en tiempo real de una red de sensores en la programación; Chang et al. (2009) formulan el problema incluyendo ventanas de tiempo y Weyland et al. (2013) hacen lo propio con tiempos límites de entrega. Por su parte, Marinakis y Marinaki (2010) consideran la situación en la que un subconjunto de los clientes debe visitarse y el número de clientes visitados es incierto, mientras que Bastian y Rinnooy Kan (1992) modelan el ruteo de vehículos estocástico.

Ampliaciones del TSP aleatorio se presentan por Tang y Miller-Hooks (2007) así como Bertazzi y Maggioni (2014). Los primeros proponen el "Problema del Vendedor Viajero Aleatorio Generalizado" donde cada cliente pertenece a un clúster, existiendo la posibilidad de que un clúster dado no requiera servicio. Los segundos analizan la localización de una planta bajo incertidumbre en la ubicación de los clientes, debiendo además decidir cómo estos se atenderán por los vendedores.

El TSP también se ha enriquecido con perspectivas multicriterio, dando lugar al TSP multiobjetivo. Esta modalidad se estudia por Florios y Mavrotas (2014) y Wang et al. (2015b), quienes usan algoritmos evolutivos, y Paquete y Stützle (2009), que lo usan como prueba en el desarrollo de algoritmos de búsqueda aleatoria local. Finalmente, Wang et al. (2015a), abordan el TSP con incertidumbre y objetivos múltiples.

La metodología aquí propuesta busca desarrollar un modelo que permita determinar cómo un conjunto de clientes potenciales debe dividirse entre un conjunto de vendedores. Esto se puede caracterizar como un problema de manejo de la fuerza de ventas que, en la medida en que toma en cuenta los costos de viaje de los vendedores, está relacionado con el TSP. Sin embargo, en contraste con los estudios previos, el elemento central de esta propuesta es la incorporación formal del conocimiento sobre las cualidades de los compradores potenciales y vendedores al modelo de la decisión, bajo una óptica de Análisis de Decisiones. Esto tiene la ventaja adicional de que las recomendaciones producidas siguen los axiomas de la toma decisión racional (Resnik, 1987).

\section{Metodología}

En esta sección se proporciona una descripción general de la metodología propuesta, proporcionando mayores detalles al trabajar un caso de estudio. Consideremos al gerente de una tienda que debe distribuir un conjunto de clientes potenciales entre sus vendedores. Se proponen cinco pasos para desarrollar un modelo para esta decisión. 
A. Establecer las cualidades de un vendedor que sean relevantes al resultado de su visita a un cliente potencial (por ejemplo, facilidad de palabra, si ha vendido antes a un cliente potencial dado, conocimiento técnico, años de experiencia, etcétera). Si alguna de estas cualidades carece de escala natural, se deberá proponer una métrica que sea lo suficientemente clara para los involucrados en el desarrollo del modelo.

B. Definir las características de los clientes potenciales que influyan el resultado de una visita a ellos y la cantidad de producto que pueden comprar. Estos factores pueden ser el tipo de empresa (local, nacional, transnacional), tamaño, si ha comprado producto antes, etcétera. Al igual que en el punto anterior, se deberán crear escalas para aquellas cualidades de los clientes potenciales que no sean medibles directamente.

C. Desarrollar un modelo para predecir el resultado de asignar un cierto vendedor a un cliente potencial dado. Dicho modelo tendrá como entrada las cualidades del cliente potencial y del vendedor y, mediante distribuciones de probabilidad condicionadas en los niveles de estas cualidades, producirá el valor esperado de la venta de esta asignación. En este trabajo, se utilizan diagramas de influencia para desarrollar el modelo.

Las distribuciones de probabilidad del modelo deben basarse, preferentemente, en registros históricos sobre el desempeño de los vendedores y las compras de los clientes potenciales. En ausencia de estos, se pueden usar distribuciones de probabilidad subjetiva elicitadas del gerente de ventas o los vendedores. En este caso, las probabilidades deben obtenerse por procedimientos que aseguren valores legítimos de probabilidad (por ejemplo, mediante la rueda de probabilidad) y se debe verificar la coherencia de las probabilidades obtenidas (Morgan y Henrion, 2004).

D. Calcular las asignaciones que maximicen la venta total esperada. Los valores esperados de la venta para todas las asignaciones posibles cliente potencial-vendedor se usan en la formulación de un problema de programación lineal entera binaria, cuyo objetivo es maximizar el valor esperado de la venta total, sujeto a un límite del número de clientes potenciales asignado a cada vendedor.

E. Para incorporar el costo de viaje de los vendedores, se calcula la ganancia neta como la venta total esperada, menos el producto de la distancia total viajada por los vendedores y un costo unitario por distancia. En este trabajo, para mantener la simplicidad, la secuencia en la que un vendedor visita a sus clientes potenciales se determina por la regla de que la visita siguiente es al cliente potencial aún no visitado, más cercano a su posición actual. Aplicando una heurística de mejora comenzando con la solución encontrada en (D), se determina el conjunto de asignaciones que maximice la ganancia neta.

\section{Desarrollo de caso de estudio}

En esta sección se ilustra la metodología trabajando un problema hipotético, donde una compañía debe decidir cómo distribuye veinte clientes potenciales entre cuatro vendedores. De forma similar a como ocurre con las ventas técnicas, una venta comienza cuando el vendedor visita al cliente potencial, sigue con este último solicitando una cotización y termina con el cierre de la venta. Este es un caso de estudio empleado con fines ilustrativos, lo que se presenta en la sección del desarrollo del caso de estudio y los resultados representa la apreciación de los autores de este trabajo sobre el proceso de ventas.

\section{Cualidades DE los Vendedores}

Los vendedores de la compañía se caracterizan en dos dimensiones:

1. Facilidad de Palabra $\left(F_{P}\right)$

2. Conocimiento Técnico $\left(C_{T}\right)$

Ambas cualidades pueden tomar los valores Alto (A) o Bajo (B).

\section{Cualidades De los Clientes potenciales}

Los clientes potenciales son empresas de la zona, que se caracterizan según:

1. Si la empresa ya ha comprado anteriormente producto a la compañía, y si, en la compra anterior, trató con alguno de los vendedores actuales.

2. Si al solicitar una cotización, la empresa especifica completamente su requerimiento (cantidad, tipo de pieza, medidas, material, etcétera) o más bien describe una necesidad y solicita al vendedor formular una cotización adecuada. Se introduce la variable "Definición de la solicitud de cotización" $\left(D_{F}\right)$ que en el primer caso es Precisa $\left(D_{F}=\mathrm{P}\right)$ y en el segundo Vaga $\left(D_{F}=\mathrm{V}\right)$. 
3. El estado de la empresa en cuanto a crecimiento $\left(E_{C}\right)$, que puede ser Crece $\left(E_{C}=C\right)$ o No Crece $\left(E_{C}=\mathrm{NC}\right)$.

4. El monto $(M)$, en dinero, que compra la empresa.

5. La localización $(x, y)$ de la empresa.

\section{Modelo del resultado de la asignación CLIENTE POTENCIAL-VENDEDOR}

Se utilizan diagramas de influencia para calcular la venta esperada de una asignación vendedor-cliente potencial dada. En estos diagramas (Figuras 1 y 2), los cuadros representan decisiones y los círculos variables inciertas (Howard y Abbas, 2015). Si una flecha va de un cuadro a un círculo, la decisión del primero afecta la distribución de probabilidad de la variable del segundo. Si una flecha conecta dos círculos, la distribución de probabilidad de la variable en la punta de la flecha está condicionada en el valor de la variable en su base.

El proceso de venta comienza con el vendedor visitando al cliente potencial, lo que puede producir una solicitud de cotización. Si esto ocurre, puede concretarse la venta o no. Se presentan dos modelos, uno para clientes potenciales que no han comprado antes y otro para los que sí.

\section{EL CLIENTE POTENCIAL NO HA COMPRADO ANTES A LA COMPAÑÍA}

El diagrama de influencia para calcular el valor esperado del monto vendido se muestra en la Figura 1, con la Tabla 1 identificando las variables. La probabilidad de que la visita del vendedor resulte en la solicitud de una cotización $\left(R_{V}=S\right)$ depende de la facilidad de palabra del vendedor $\left(F_{P}\right)$ y el estado de crecimiento de la empresa $\left(E_{C}\right)$, por lo que está condicionada en ambos factores $p_{R V}\left(R_{V} \mid E_{C}, F_{P}\right)$. Estas probabilidades deben reflejar el hecho de que es más probable obtener una solicitud de

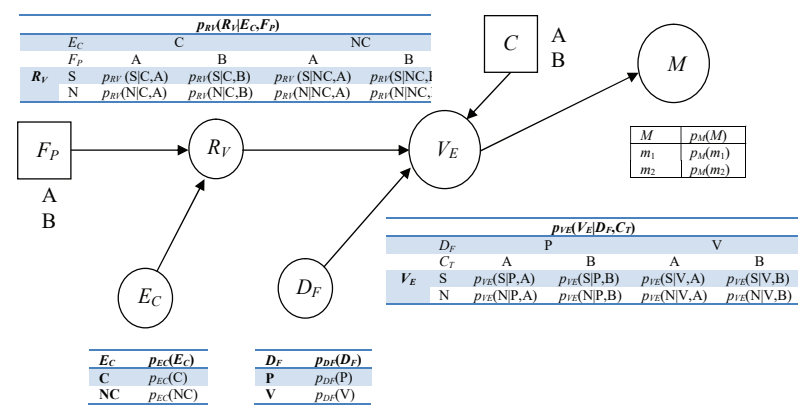

Figura 1. Diagrama de influencia del resultado de la asignación cliente potencial-vendedor para clientes potenciales que no han comprado anteriormente cotización si el vendedor tiene mayor facilidad de palabra y la empresa crece.

Si se solicita una cotización $\left(R_{v}=S\right)$, esta se concretará en una venta $\left(V_{E}=S\right)$ con probabilidad $p_{V E}\left(V_{E} \mid D_{H} C_{T}\right)$ condicionada en la definición de la solicitud de cotización $\left(D_{F}\right)$ y el conocimiento técnico del vendedor $\left(C_{T}\right)$. Si la solicitud de cotización se especifica con precisión $\left(D_{F}=P\right)$, esto es señalando cantidades, medidas, materiales, etcétera, será más fácil producir una cotización satisfactoria. Si, en cambio, la solicitud no especifica algunos elementos $\left(D_{F}=\mathrm{V}\right)$ y pide al vendedor que haga recomendaciones sobre ellos, dependerá del conocimiento técnico de este último producir una cotización aceptable.

Finalmente, si se concreta la venta $\left(V_{E}=S\right)$, el monto vendido $(M)$ al cliente potencial toma los valores $m_{1}$ y $m_{2}$ con probabilidades $p_{M}\left(m_{1}\right)$ y $p_{M}\left(m_{2}\right)$, respectivamente.

$\mathrm{Al}$ asignar un cliente potencial a un vendedor con $F_{P}$ y $C_{T}$ dados, el valor esperado de la venta a este cliente potencial, $\mathrm{E}\left[M \mid F_{P}, C_{T}\right]$ se calcula mediante las ecuaciones 1 a 6 , en las que la notación $E_{\text {NODO }}$ y $p_{\text {NODO }}$ se refiere, respectivamente, al valor esperado y la distribución de probabilidad del círculo $(N O D O)$ correspondiente de la Figura 1.

$E_{M}[M]=m_{1} \times p_{M}\left(m_{1}\right)+m_{2} \times p_{M}\left(m_{2}\right)$

$E_{V E}\left[M \mid D_{F}=\mathrm{P}, C_{T}\right]=p_{V E}\left(\mathrm{~S} \mid \mathrm{P}, C_{T}\right) \times E_{M}[M]$

$E_{V E}\left[M \mid D_{F}=\mathrm{V}, C_{T}\right]=p_{V E}\left(\mathrm{~S} \mid \mathrm{V}, \mathrm{C}_{T}\right) \times E_{M}[M]$

$E_{D F}\left[M \mid C_{T}\right]=p_{D F}(\mathrm{P}) \times E_{V E}\left[M \mid D_{F}=\mathrm{P}, C_{T}\right]+p_{D F}(\mathrm{~V}) \times$ $E_{V E}\left[M \mid D_{F}=\mathrm{V}, C_{T}\right]$

$p\left(R_{V}=S \mid F_{P}\right)=p_{E C}(\mathrm{C}) \times p_{R V}\left(\mathrm{~S} \mid \mathrm{C}, F_{P}\right)+p_{E C}(\mathrm{NC}) \times p_{R V}\left(\mathrm{~S} \mid \mathrm{NC}, F_{P}\right)$

$\mathrm{E}\left[M \mid F_{P \prime} C_{T}\right]=E_{R V}\left[M \mid F_{P \prime} C_{T}\right]=p\left(R_{V}=S \mid F_{P}\right) \times E_{D F}\left[M \mid C_{T}\right]$

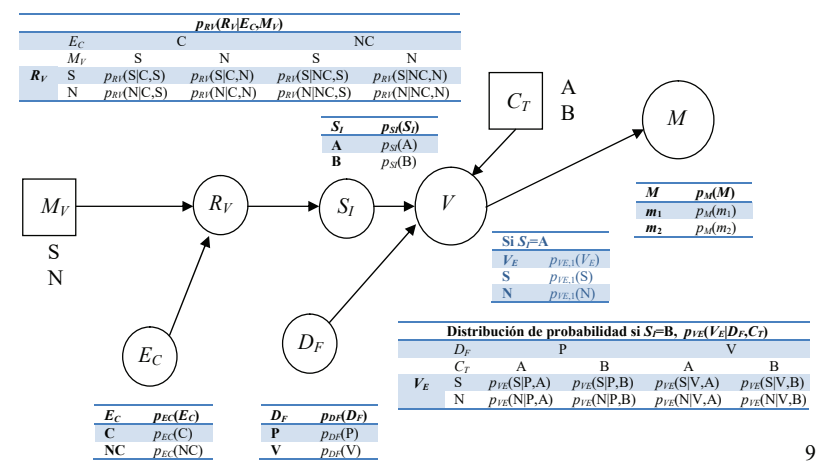

Figura 2. Diagrama de influencia del resultado de la asignación cliente potencial-vendedor para clientes potenciales que han comprado anteriormente 
Tabla 1. Definición de variables en el diagrama de influencia de la Figura 1

\begin{tabular}{|c|c|c|}
\hline Variable & Nombre & Valores Posibles \\
\hline$F_{p}$ & Facilidad de palabra del vendedor & $\begin{array}{l}\text { A: Alta } \\
\text { B: Baja }\end{array}$ \\
\hline$C_{T}$ & Conocimiento Técnico del vendedor & $\begin{array}{l}\text { A: Alto } \\
\text { B: Bajo }\end{array}$ \\
\hline$E_{C}$ & Estado de crecimiento del cliente potencial & $\begin{array}{l}\text { C:Crece } \\
\text { NC: No crece }\end{array}$ \\
\hline$R_{V}$ & Resultado de la visita del vendedor al cliente potencial & $\begin{array}{l}\text { S: El cliente potencial solicita cotización } \\
\mathrm{N} \text { : El cliente potencial no solicita cotización }\end{array}$ \\
\hline$D_{F}$ & Definición de la solicitud de cotización & $\begin{array}{l}\text { P: Precisa } \\
\text { V: Vaga }\end{array}$ \\
\hline$V_{E}$ & Venta Lograda & $\begin{array}{l}\text { S: Se concreta la venta } \\
\text { N: No se concreta la venta }\end{array}$ \\
\hline$M$ & Monto vendido & $m_{1}$ y $m_{2}$ \\
\hline
\end{tabular}

\section{EL CLIENTE POTENCIAL YA HA COMPRADO ANTES A LA COMPAÑÍA}

En este caso el modelo debe reflejar que, si se asigna a este cliente potencial un vendedor al que ya le ha comprado antes, es más probable conseguir una solicitud de cotización y, si esta es similar a la de ventas anteriores, se facilita la formulación de la cotización. El diagrama de influencia para este caso se muestra en la Figura 2, con la Tabla 2, mostrando la definición de las variables no definidas en la Tabla 1. La variable $M_{V}$ " "Mismo Vendedor" vale "S" si el vendedor asignado es el que cerró la venta anterior, $\mathrm{y}$ " $\mathrm{N}$ " si es un vendedor distinto. El valor de $M_{V}$ y Estado de Crecimiento $\left(E_{C}\right)$ condicionan las probabilidades $p_{R V}\left(R_{V} \mid E_{C^{\prime}} M_{V}\right)$ del Resultado de la Visita $\left(R_{V}\right)$.

$\mathrm{Si}$ el resultado de la visita del vendedor es la solicitud de una cotización $\left(R_{V}=S\right)$, la variable $S_{I}$ indica qué tan técnicamente similar es lo planteado en esta solicitud, respecto a las ventas completadas anteriormente. Si la nueva solicitud es técnicamente similar a lo comprado por este cliente en ocasiones previas $\left(S_{I}=\mathrm{A}\right)$, la probabilidad de cerrar la venta es $p_{V E, 1}(\mathrm{~S})$. Si, por el contrario, la solicitud no guarda parecido a pedidos anteriores $\left(S_{I}=\mathrm{B}\right)$, la probabilidad de cerrar la venta depende de la definición de la solicitud de cotización y el conocimiento técnico del vendedor.
La evaluación de la Figura 2 permite calcular el valor esperado del monto vendido, $\mathrm{E}\left[M \mid M_{V} C_{T}\right]$, de acuerdo con las ecuaciones 7 a 14 .

$$
\begin{aligned}
& E_{M}[M]=m_{1} \times p_{M}\left(m_{1}\right)+m_{2} \times p_{M}\left(m_{2}\right) \\
& E_{V E}\left[M \mid S_{I}=\mathrm{A}\right]=p_{V E, 1}(\mathrm{~S}) \times E_{M}[M] \\
& E_{V E}\left[M \mid S_{I}=\mathrm{B}, D_{F}=\mathrm{P}, C_{T}\right]=p_{V E}\left(\mathrm{~S} \mid \mathrm{P}, C_{T}\right) \times \mathrm{E}_{M}[M] \\
& E_{V E}\left[M \mid S_{I}=\mathrm{B}, D_{F}=\mathrm{V}, C_{T}\right]=p_{V E}\left(\mathrm{~S} \mid \mathrm{V}, C_{T}\right) \times \mathrm{E}_{M}[M] \\
& E_{D F}\left[M \mid S_{I}=\mathrm{B}, C_{T}\right]=p_{D F}(\mathrm{P}) \times E_{V E}\left[M \mid S_{I}=\mathrm{B}, D_{F}=\mathrm{P}, C_{T}\right]+p_{D F}(\mathrm{~V}) \times \\
& E_{V E}\left[M \mid S_{I}=\mathrm{B}, D_{F}=\mathrm{V}, C_{T}\right] \\
& E_{S I}\left[M \mid C_{T}\right]=p_{S I}(\mathrm{~A}) \times E_{V E}\left[M \mid S_{I}=\mathrm{A}\right]+p_{S I}(\mathrm{~B}) \times \mathrm{E}_{D F}\left[M \mid S_{I}=\mathrm{B}, C_{T}\right]
\end{aligned}
$$

\begin{tabular}{|c|c|c|}
\hline Variable & Nombre & Valores Posibles \\
\hline$M_{V}$ & Mismo Vendedor & $\begin{array}{l}\text { S: El vendedor asignado es el mismo que cerró la } \\
\text { venta anterior } \\
\text { N: El vendedor asignado es distinto al que cerró la } \\
\text { venta anterior }\end{array}$ \\
\hline$S_{I}$ & $\begin{array}{l}\text { Grado de semejanza entre la solicitud de } \\
\text { cotización actual y la cubierta en ventas previas }\end{array}$ & $\begin{array}{l}\text { A: Alta } \\
\text { B: Baja }\end{array}$ \\
\hline
\end{tabular}$$
p\left(R_{V}=\mathrm{S} \mid M_{V}\right)=p_{E C}(\mathrm{C}) \times p_{R V}\left(\mathrm{~S} \mid \mathrm{C}, M_{V}\right)+
$$$$
p_{E C}(\mathrm{NC}) \times p_{R V}\left(\mathrm{~S} \mid \mathrm{NC}, \mathrm{M}_{V}\right)
$$

$\mathrm{E}\left[M \mid M_{V} C_{T}\right]=\mathrm{E}_{R V}\left[M \mid M_{V} C_{T}\right]=p\left(R_{V}=S \mid M_{V}\right) \times E_{S L}\left[M \mid C_{T}\right]$

Tabla 2. Definición de variables adicionales en el diagrama de influencia de la Figura 2 
DETERMINACIÓN DE LAS ASIGNACIONES QUE MAXIMIZAN LA VENTA TOTAL ESPERADA

Sea $E(i, j)$ el valor esperado de la venta si se asigna el cliente potencial $i$ al vendedor $j$. El cálculo de $\mathrm{E}(i, j)$ depende de si el cliente potencial ha comprado antes producto a la compañía o no:

$\mathrm{E}(i, j)=\mathrm{E}\left[M \mid F_{p}, C_{T}\right]$

Si el cliente potencial $i$ no ha comprado producto anteriormente

$\mathrm{E}(i, j)=\mathrm{E}\left[M \mid M_{V}, C_{T}\right]$

Si el cliente potencial $i$ ha comprado producto anteriormente

Donde $F_{P}$ y $C_{T}$ son, respectivamente, la facilidad de palabra y el conocimiento técnico del vendedor $j \mathrm{y}$, si el cliente potencial $i$ ha comprado anteriormente, la variable $M_{V}$ indica si la venta previa fue cerrada por dicho vendedor. De igual forma, las distribuciones de probabilidad usadas en el cálculo de los valores esperados $\mathrm{E}\left[M \mid F_{p}, C_{T}\right]$ y $\mathrm{E}\left[M \mid M_{V} C_{T}\right]$ (ecuaciones 1-6 y 7-14, respectivamente) están dadas por el cliente potencial $i$ que se trate. Si $X(i, j)$ toma el valor 1 cuando el cliente potencial $i$ se asigna al vendedor $j$ y cero si no, la venta total esperada $\left(M_{T}\right)$ de un conjunto de asignaciones, en un problema con $N_{V}$ vendedores y $N_{C P}$ clientes potenciales, se calcula como

$$
M_{T}=\sum_{i=1}^{N_{C P}} \sum_{j=1}^{N_{V}} E(i, j) \times X(i, j)
$$

Introduciendo las restricciones de que cada cliente potencial se asigne a un solo vendedor y que a cada vendedor se le asigne un máximo de $N_{C P, M A X}$ clientes potenciales, y calculando previamente los valores $E(i, j)$ $\left(i=1, \ldots, N_{C P}, j=1, \ldots, N_{V}\right)$ según las ecuaciones (15) y (16), las asignaciones que maximizan $M_{T}$ se determinan solucionando el problema de programación lineal entera

$$
\max _{X(i, j)}\left[M_{T}=\sum_{i=1}^{N_{C P}} \sum_{j=1}^{N_{V}} E(i, j) \times X(i, j)\right]
$$

Sujeto a

$$
\begin{aligned}
& \sum_{j=1}^{N_{V}} X(i, j)=1 \quad \text { para } i=1, \ldots, N_{C P} \\
& \sum_{i=1}^{N_{C P}} X(i, j) \leq N_{C P, M A X} \quad \text { para } j=1, \ldots, N_{V} \\
& \text { y } X(i, j)=0 \text { ó } 1
\end{aligned}
$$

Sea $C_{j}$ el conjunto de clientes potenciales asignados al vendedor $j$, en otras palabras

$C_{j}=\{i \mid X(i, j)=1\} \quad j=1, \ldots, N_{V}$

La solución al problema lineal de las ecuaciones (18) a (20), proporciona los conjuntos $C_{1}, C_{2}, \ldots, C_{N V}$ que maximizan la venta total esperada $M_{T}$.

\section{DETERMINACIÓN DE LAS ASIGNACIONES INCLUYENDO COSTOS DE TRANSPORTE}

Sea $D\left(C_{j}\right)$ la distancia total viajada por el vendedor $j$ al visitar a los clientes potenciales del conjunto $C_{j}$ y regresar a la tienda. En este trabajo, la secuencia de visitas a los miembros de $C_{j}$ se determina suponiendo que, al terminar una visita, el vendedor pasa al cliente potencial, aun no visitado, más cercano a su posición actual. Sumando las distancias totales viajadas por los vendedores, e introduciendo un costo unitario por distancia viajada $\left(c_{D}\right)$, la ganancia neta $G_{N}$ se determina como

$$
G_{N}=\sum_{i=1}^{N_{C P}} \sum_{j=1}^{N_{V}} E(i, j) \times X(i, j)-c_{D} \sum_{j=1}^{N_{V}} D\left(C_{j}\right)
$$

Donde $C_{j}=\{i \mid X(i, j)=1\}$. Para encontrar un diseño aceptable incluyendo los costos de transporte, se propone tomar como solución inicial los conjuntos encontrados al solucionar el problema de programación lineal de la sección "Determinación de las asignaciones que maximizan la venta total esperada" y aplicar una heurística incremental: se prueban intercambios entre los miembros de los conjuntos $C_{j}\left(j=1, \ldots, N_{V}\right)$ y, si esto resulta en un incremento de $G_{N}$ el intercambio se hace permanente. De forma más precisa:

1. Dados los conjuntos $C_{j}$ obtenidos resolviendo el problema de programación lineal de la sección "Determinación de las asignaciones que maximizan la venta total esperada", calcular el valor $G_{N}{ }^{*}$ como el valor de $G_{N}$ para estas asignaciones. Hacer $j=1, i=j+1$.

2. Intercambiar el valor $k \in C_{j}$ con el valor $l \in C_{i}$ (hacer $X(k, j)=X(l, i)=0$ y $X(l, j)=X(k, i)=1)$ y calcular $G_{N}$. Si $G_{N}>$ $G_{N}{ }^{*}$ hacer este intercambio permanente y sustituir $G_{N}{ }^{*}=G_{N}$. Repetir hasta haber probado el intercambio de todos los elementos de $C_{j}$ con todos de $C_{i}$.

3. Si $i<N_{V}$ entonces hacer $i=i+1$ y regresar al paso 2 . Si $i=N_{V}$ y $j<N_{V}-1$ entonces hacer $j=j+1, i=j+1$ e ir al paso 2 .

\section{Resultados del caso de estudio}

En esta sección se concluye la ilustración de la aplicación de la metodología; para ello, se asignan valores a los pa- 
rámetros del modelo, que se incluyen en las Tablas 3 a 8 . Estos valores tienen fines ilustrativos; sin embargo, para una aplicación de la vida real, deberá verificarse que los valores numéricos asignados a parámetros y probabilidades representan fielmente el conocimiento de la persona responsable de la decisión (Lindley, 2006).

Las cualidades de los vendedores se indican en la Tabla 3. La Tabla 4 muestra la localización de los clientes potenciales y las distribuciones de probabilidad del monto de compra $(M)$, estado de crecimiento $\left(E_{C}\right)$ y definición de la solicitud de cotización $\left(D_{F}\right)$. La columna "Compra Previa", indica si un cliente potencial ha comprado producto anteriormente.

La Tabla 5 muestra el vendedor que cerró la última venta con el cliente potencial, la probabilidad de que una solicitud de cotización sea similar a la despachada anteriormente $p_{S I}(\mathrm{~A}) \mathrm{y}$, de ser el caso, la probabilidad de cerrar la venta $p_{V E, 1}(\mathrm{~S})$. La ausencia de un valor en la primera columna, indica que este cliente potencial trató anteriormente con un vendedor no considerado actualmente.

La Figura 3 muestra con marcadores, rellenos para los clientes potenciales que han comprado antes y vacíos para los que no, y de tamaño proporcional a $\mathrm{E}_{M}[M]$, la localización de los clientes potenciales. La notación $V_{A}=k$ indica que el vendedor $k$ se encargó de la venta anterior.

La distribución de probabilidad del resultado de la visita $\left(R_{V}\right)$ se muestra en la Tabla 6 para clientes potenciales que no han comprado antes y en la Tabla 7 para aquellos que sí lo han hecho. La Tabla 8 muestra la probabilidad de cerrar la venta, $p_{V E}\left(V_{E} \mid D_{F}, C_{T}\right)$, cuando se tiene la solicitud de cotización de un cliente potencial que no ha comprado antes o de uno que, habiendo comprado previamente, presenta una solicitud de cotización técnicamente distinta a las antes surtidas.

Tabla 3. Cualidades de los vendedores

\begin{tabular}{ccc}
\hline Vendedor & Facilidad de Palabra $\left(F_{P}\right)$ & Conocimiento Técnico $\left(C_{T}\right)$ \\
\hline 1 & B & A \\
2 & A & B \\
3 & B & B \\
4 & A & A \\
\hline
\end{tabular}

Tabla 4. Localización y cualidades de los clientes potenciales

\begin{tabular}{ccccccccc}
\hline $\begin{array}{c}\text { Cliente } \\
\text { potencial }\end{array}$ & $x$ & $y$ & $m_{1}(\$)$ & $m_{2}(\$)$ & $p_{M}\left(m_{1}\right)$ & $p_{E C}(\mathrm{C})$ & $p_{D F}(\mathrm{P})$ & Compra Previa \\
\hline 1 & 8 & 16 & 200 & 70 & 0.53 & 0.11 & 0.5 & No \\
2 & 10 & 29 & 250 & 50 & 0.92 & 0.90 & 0.8 & Si \\
3 & 19 & 28 & 520 & 380 & 0.53 & 0.30 & 0.8 & No \\
4 & 10 & 43 & 450 & 350 & 0.41 & 0.68 & 0.1 & No \\
5 & 11 & 46 & 750 & 600 & 0.77 & 0.95 & 0.6 & No \\
6 & 21 & 40 & 600 & 270 & 0.83 & 0.02 & 0.6 & Si \\
7 & 41 & 30 & 500 & 250 & 0.46 & 0.98 & 1.0 & No \\
8 & 41 & 38 & 840 & 520 & 0.50 & 0.58 & 0.5 & No \\
9 & 58 & 16 & 610 & 400 & 0.71 & 0.40 & 0.9 & Si \\
10 & 65 & 5 & 470 & 400 & 0.43 & 0.10 & 0.3 & Si \\
11 & 58 & 38 & 500 & 450 & 0.37 & 0.28 & 0.2 & No \\
12 & 28 & 63 & 180 & 150 & 0.94 & 0.10 & 0.6 & No \\
13 & 68 & 26 & 800 & 650 & 0.35 & 0.16 & 0.7 & Si \\
14 & 71 & 30 & 820 & 610 & 0.71 & 0.80 & 0.6 & Si \\
15 & 2 & 79 & 450 & 390 & 0.67 & 0.16 & 1.0 & Si \\
16 & 10 & 92 & 790 & 590 & 0.70 & 0.28 & 0.3 & Si \\
17 & 33 & 95 & 750 & 620 & 0.30 & 0.65 & 0.2 & No \\
20 & 100 & 91 & 190 & 120 & 0.28 & 0.30 & 0.3 & 0.5 \\
\end{tabular}


Tabla 5. Cualidades de los clientes potenciales que han comprado producto anteriormente

\begin{tabular}{cccc}
\hline $\begin{array}{c}\text { Cliente } \\
\text { potencial }\end{array}$ & $\begin{array}{c}\text { Vendedor que realizó la } \\
\text { venta anterior }\end{array}$ & $p_{S I}(\mathrm{~A})$ & $p_{V E, 1}(\mathrm{~S})$ \\
\hline 2 & --- & 0.6 & 0.8 \\
6 & 2 & 0.3 & 0.7 \\
9 & 2 & 0.7 & 0.9 \\
11 & 3 & 0.25 & 0.8 \\
14 & --- & 0.5 & 0.8 \\
15 & 1 & 0.6 & 0.9 \\
16 & 4 & 0.4 & 0.8 \\
17 & 3 & 0.63 & 0.9 \\
19 & --- & 0.25 & 0.8 \\
20 & --- & 0.21 & 0.9 \\
\hline
\end{tabular}

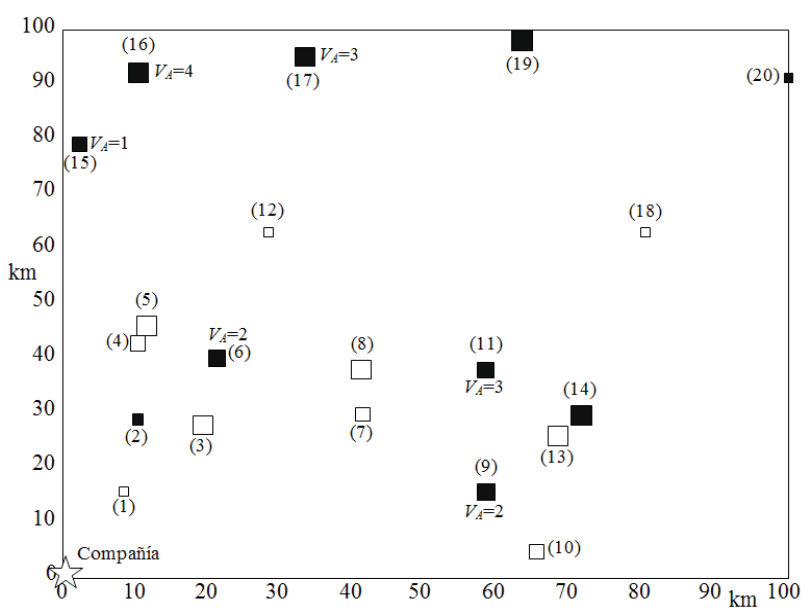

Figura 3. Localización de los clientes potenciales

La Tabla 9 muestra los valores de $E(i, j)$ para todas las asignaciones posibles de vendedores y clientes potenciales.

Restringiendo un máximo de cinco clientes potenciales por vendedor $\left(N_{C P, M A X}=5\right)$, la solución del problema de programación lineal entera descrito en la sección "Determinación de las asignaciones que maximizan la venta total esperada" produce las asignaciones óptimas mostradas en la Tabla 10, donde se utiliza la notación $C_{j}$ para referirse al conjunto de clientes potenciales asignados al vendedor $j$ y se incluye la venta esperada del vendedor $j,\left(E_{j}\right)$. Estas asignaciones producen una venta total esperada $\left(M_{T}\right)$ de $\$ 3897.1$.

Para las asignaciones de la Tabla 10, las distancias calculadas según lo descrito en la sección "Determinación de las asignaciones incluyendo costos de transporte" se muestran en la Tabla 11, mientras la Figura 4 ilustra las trayectorias seguidas por los vendedores; $D_{T}$ es la distancia total viajada por los vendedores.

Para incorporar los costos de viaje, se calcula la ganancia neta de un conjunto asignaciones $G_{N}$ como la ecuación (22) y se aplica la heurística descrita en la sección "Determinación de las asignaciones incluyendo costos de transporte", tomando como punto de inicio las asignaciones de la Tabla 10. Para un costo de viaje de $c_{D}=1 \$ / \mathrm{km}$, la Tabla 12 muestra la asignación proporcionada por la heurística. La Figura 5 muestra que la asignación encontrada suaviza la ruta del vendedor 2 y acorta la del vendedor 3. Sin embargo, mantiene sin cambios las asignaciones del vendedor 4, quien produce la mayor contribución a la venta total.

Tabla 6. Valores de $p_{R V}\left(R_{V} \mid E_{C}, F_{P}\right)$

\begin{tabular}{cccccc}
\hline & $E_{C}$ & \multicolumn{2}{c}{$\mathrm{C}$} & \multicolumn{2}{c}{$\mathrm{NC}$} \\
\hline & $F_{P}$ & $\mathrm{~A}$ & $\mathrm{~B}$ & $\mathrm{~A}$ & $\mathrm{~B}$ \\
$R_{V}$ & $\mathrm{~S}$ & 0.9 & 0.6 & 0.3 & 0.1 \\
& $\mathrm{~N}$ & 0.1 & 0.4 & 0.7 & 0.9
\end{tabular}

\begin{tabular}{cccccc}
\multicolumn{6}{c}{ Tabla 7. Valores de $p_{R V}\left(R_{V} \mid E_{C} M_{V}\right)$} \\
\hline$E_{C}$ & \multicolumn{3}{c}{$\mathrm{C}$} & \multicolumn{3}{c}{$\mathrm{NC}$} \\
\hline & $M_{V}$ & $\mathrm{~S}$ & $\mathrm{~N}$ & $\mathrm{~S}$ & $\mathrm{~N}$ \\
$R_{V}$ & $\mathrm{~S}$ & 0.9 & 0.7 & 0.4 & 0.1 \\
& $\mathrm{~N}$ & 0.1 & 0.3 & 0.6 & 0.9 \\
\hline
\end{tabular}

Tabla 8. Valores de $p_{V E}\left(V_{E} \mid D_{F} C_{T}\right)$

\begin{tabular}{ccccccc}
\hline & $D_{F}$ & \multicolumn{2}{c}{$\mathrm{P}$} & \multicolumn{2}{c}{$\mathrm{V}$} \\
\hline & $\mathrm{C}_{\mathrm{T}}$ & $\mathrm{A}$ & $\mathrm{B}$ & $\mathrm{A}$ & $\mathrm{B}$ \\
$V_{E}$ & $\mathrm{~S}$ & 0.9 & 0.7 & 0.8 & 0.2 \\
& $\mathrm{~N}$ & 0.1 & 0.3 & 0.2 & 0.8 \\
\hline
\end{tabular}


Tabla 9. Valores de $E(i, j)$ (en \$) cuando se asigna el vendedor $j$ al cliente potencial $i$

\begin{tabular}{|c|c|c|c|c|}
\hline \multirow{2}{*}{$\begin{array}{c}\text { Cliente Potencial } \\
i\end{array}$} & \multicolumn{4}{|c|}{ Vendedor $(j)$} \\
\hline & 1 & 2 & 3 & 4 \\
\hline 1 & 18 & 23 & 10 & 43 \\
\hline 2 & 124 & 107 & 107 & 124 \\
\hline 3 & 100 & 134 & 70 & 193 \\
\hline 4 & 138 & 63 & 39 & 222 \\
\hline 5 & 353 & 308 & 203 & 534 \\
\hline 6 & 48 & 124 & 33 & 48 \\
\hline 7 & 193 & 225 & 149 & 291 \\
\hline 8 & 223 & 191 & 114 & 372 \\
\hline 9 & 168 & 273 & 155 & 168 \\
\hline 10 & 54 & 54 & 23 & 128 \\
\hline 11 & 102 & 54 & 110 & 102 \\
\hline 12 & 23 & 33 & 14 & 56 \\
\hline 13 & 110 & 152 & 69 & 242 \\
\hline 14 & 366 & 291 & 291 & 366 \\
\hline 15 & 186 & 69 & 69 & 76 \\
\hline 16 & 161 & 103 & 103 & 323 \\
\hline 17 & 278 & 219 & 324 & 278 \\
\hline 18 & 17 & 18 & 7 & 45 \\
\hline 19 & 209 & 137 & 137 & 209 \\
\hline 20 & 34 & 26 & 26 & 34 \\
\hline
\end{tabular}

Tabla 10. Asignaciones que maximizan la venta total esperada

\begin{tabular}{ccc}
\hline Vendedor $(j)$ & Clientes potenciales asignados $C_{j}$ & Venta esperada del vendedor $E_{j}$ \\
\hline 1 & $\{2,10,14,15,19\}$ & $\$ 939.6$ \\
2 & $\{3,6,7,9,12\}$ & $\$ 787.5$ \\
3 & $\{1,11,18,17,20\}$ & $\$ 477.2$ \\
4 & $\{4,5,8,13,16\}$ & $\$ 1692.8$ \\
\hline
\end{tabular}

Tabla 11. Distancias viajadas por los vendedores en la asignación mostrada en la Tabla 10

\begin{tabular}{|c|c|c|c|}
\hline Vendedor $(j)$ & $C_{j}($ en orden de visita $)$ & $D\left(C_{j}\right)(\mathrm{km})$ & $E_{j}$ \\
\hline 1 & $\{2,15,19,14,10\}$ & 304.6 & $\$ 939.6$ \\
\hline 2 & $\{3,6,7,9,12\}$ & 215.1 & $\$ 787.5$ \\
\hline 3 & $\{1,11,18,20,17\}$ & 307.9 & $\$ 477.2$ \\
\hline \multirow[t]{2}{*}{4} & $\{4,5,8,13,16\}$ & 288.3 & $\$ 1692.8$ \\
\hline & & 1116.2 & $\$ 3897.1$ \\
\hline
\end{tabular}


La Tabla 13 muestra las asignaciones proporcionadas por la heurística para un valor de $c_{D}=2 \$ / \mathrm{km}$. La Figura 6 permite ver que en este caso, el costo de transporte justifica modificar las asignaciones del vendedor 4 para suavizar su trayectoria.

\section{Conclusiones}

En este trabajo se presenta una metodología para abordar la decisión, relevante al manejo de la fuerza de ventas de las comercializadoras, de establecer cómo un conjunto de clientes potenciales debe dividirse entre los vendedores disponibles. La metodología toma una perspectiva de Análisis de Decisiones y tiene como principal objetivo incorporar formalmente a la decisión la información sobre vendedores y clientes potenciales que se tenga a la mano. Si bien los gerentes de ventas experimentados toman en cuenta, de forma intuitiva, a sus vendedores y clientes potenciales al tomar esta decisión, la propuesta del trabajo es caracterizar cuantita-

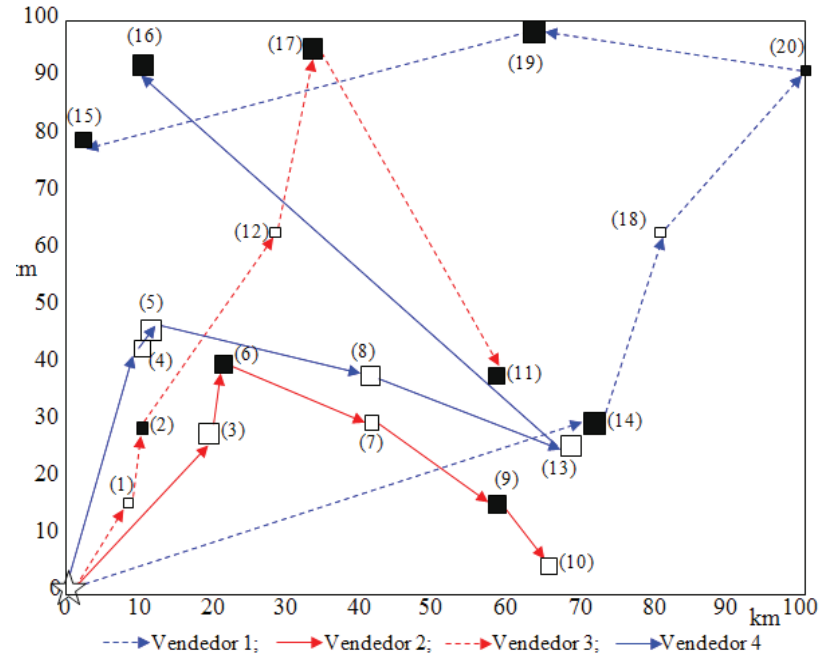

Figura 5. Ruta de los vendedores en la solución mejorada para $C_{D}=1 \$ / \mathrm{km}$

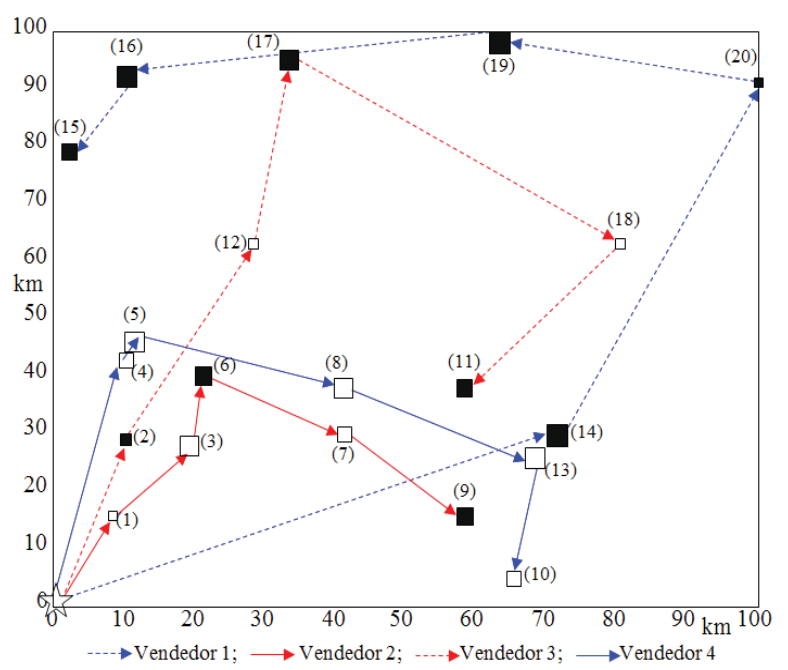

Figura 4. Ruta de los vendedores para la asignación mostrada en Tabla 10

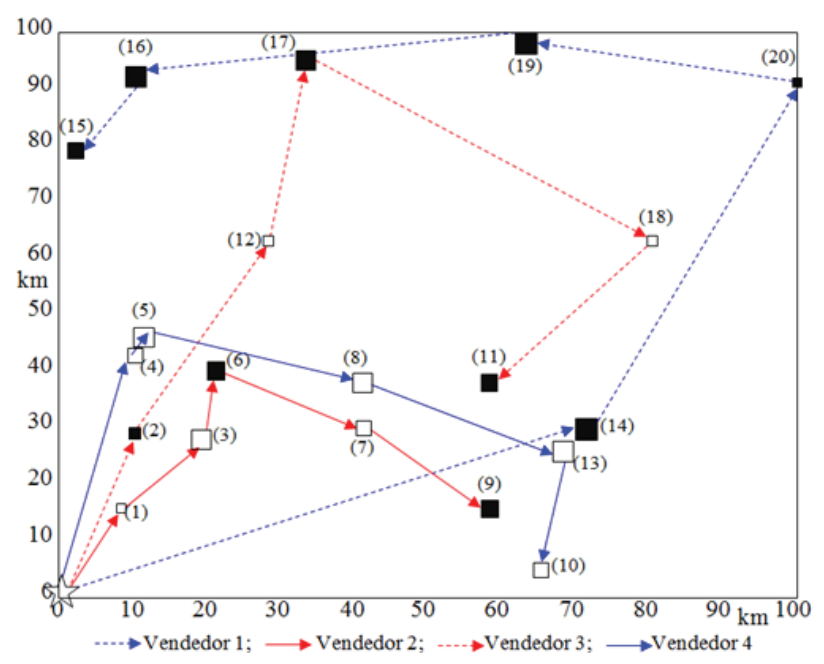

Figura 6. Ruta de los vendedores en la solución mejorada para $C_{D}=2 \$ / \mathrm{km}$

Tabla 12. Distancias viajadas por los vendedores en asignaciones mejoradas con $C_{D}=1 \$ / \mathrm{km}$

\begin{tabular}{|c|c|c|c|}
\hline Vendedor $(j)$ & $C_{j}$ (en orden de visita) & $D\left(C_{j}\right)(\mathrm{km})$ & $E_{j}$ \\
\hline 1 & $\{14,18,20,19,15\}$ & 326.3 & $\$ 812.7$ \\
\hline 2 & $\{3,6,7,9,10\}$ & 168.6 & $\$ 808.6$ \\
\hline 3 & $\{1,2,12,17,11\}$ & 233.5 & $\$ 564.8$ \\
\hline \multirow[t]{3}{*}{4} & $\{4,5,8,13,16\}$ & 288.3 & $\$ 1692.8$ \\
\hline & & 1016.7 & $\$ 3878.9$ \\
\hline & & & $\$ 2862.2$ \\
\hline
\end{tabular}


Tabla 13. Distancias viajadas por los vendedores en asignaciones mejoradas con $c_{D}=2 \$ / \mathrm{km}$

\begin{tabular}{|c|c|c|c|c|c|}
\hline Vendedor $(j)$ & $C_{j}($ En orden de visita $)$ & \multicolumn{2}{|c|}{$D\left(C_{j}\right)(\mathrm{km})$} & \multicolumn{2}{|c|}{$E_{j}$} \\
\hline 1 & $\{14,20,19,16,15\}$ & \multicolumn{2}{|c|}{329.9} & \multicolumn{2}{|c|}{$\$ 957.2$} \\
\hline 2 & $\{1,3,6,7,9\}$ & \multicolumn{2}{|c|}{150.9} & \multicolumn{2}{|c|}{$\$ 777.9$} \\
\hline 3 & $\{2,12,17,18,11\}$ & \multicolumn{2}{|c|}{261.0} & \multicolumn{2}{|c|}{$\$ 561.9$} \\
\hline \multirow[t]{3}{*}{4} & $\{4,5,8,13,10\}$ & \multicolumn{2}{|c|}{194.3} & \multicolumn{2}{|c|}{ \$ 1498.4} \\
\hline & & $D_{T}$ & 936.1 & $M_{T}$ & $\$ 3795.4$ \\
\hline & & & & $G_{N}$ & $\$ 1923.2$ \\
\hline
\end{tabular}

tivamente esta información e incluirla a un modelo de decisión.

Dada la naturaleza inherentemente impredecible de las ventas, el modelo incluirá parámetros sobre los que se tiene incertidumbre y sobre los que deberán definirse distribuciones de probabilidad con anchura adecuada al nivel de desconocimiento que se tenga. Se propone que la construcción del modelo se realice mediante diagramas de influencia, pues estos permiten mostrar gráficamente las dependencias probabilísticas entre los parámetros inciertos y las decisiones.

Se aplicó la metodología a un problema hipotético con veinte clientes potenciales y cuatro vendedores. Las características de clientes potenciales y vendedores y los valores numéricos de los parámetros y distribuciones de probabilidad presentados, tienen fines ilustrativos. Sin embargo, si un gerente de ventas de la vida real no juzga equivalentes entre sí a sus vendedores y clientes potenciales, puede aplicar la metodología aquí presentada, construyendo métricas que precisen las diferencias percibidas y definiendo distribuciones de probabilidad que representen su apreciación sobre su efecto en las ventas probables. Debe enfatizarse que si las distribuciones de probabilidad se derivan de la opinión del gerente o vendedores, estas deberán obtenerse por un procedimiento que garantice que los números proporcionados sean realmente probabilidades y será necesario realizar pruebas de coherencia sobre el conjunto de probabilidades obtenido.

\section{RefERenCIAS}

Bastian C., Rinnooy-Kan A.H.G. The stochastic vehicle routing problem revisited. European Journal of Operational Research, volumen 56 (número 3), 1992: 407-412.

Bertazzi L., Maggioni F. The stochastic capacitated traveling salesmen location problem: A computational comparison for a United States instance. Procedia - Social and Behavioral Sciences, volume 108, 2014: 47-56.
Chang T.S., Wan Y.W., Ooi W.T. A stochastic dynamic traveling salesman problem with hard time windows. European Journal of Operational Research, volumen 198 (número 3), 2009:748-759.

Chun Y.H. Multi-attribute sequential decision problem with optimizing and satisficing attributes. European Journal of Operational Research, volumen 243 (número 1), 2015: 224-232.

Cravens D.W., LaForge R.W. Salesforce deployment analysis. Industrial Marketing Management, volumen 12 (número 3), 1983: 179-192.

Da Silva R.V., Davies G., Naudé P. Assessing customer orientation in the context of buyer/supplier relationships using judgmental modeling. Industrial Marketing Management, volumen 31 (número 3), 2002: 241-252.

Edwards W., Miles R.F., Von-Winterfeldt D. Advances in Decision Analysis, New York, Cambridge University Press, 2007, pp. 13-32.

Falagario M., Sciancalepore F., Costantino N., Pietroforte R. Using a DEA-cross efficiency approach in public procurement tenders. European Journal of Operational Research, volumen 218 (número 2), 2012: 523-529.

Florios K., Mavrotas G. Generation of the exact Pareto set in Multi-Objective Traveling Salesman and Set Covering Problems. Applied Mathematics and Computing, volumen 237, 2014: 1-19.

Howard R.A. Decision Analysis: Practice and promise. Management Science, volumen 34 (número 6), 1988: 679-695.

Howard R.A. y Abbas A.E. Foundations of Decision Analysis, Boston, Pearson, 2015, pp. 123-141.

Johnston W.J. y Cooper M. Analyzing the industrial salesforce selection process. Industrial Marketing Management, volumen 10 (número 2), 1981: 139-147.

Kar A.K. A hybrid group decision support system for supplier selection using analytic hierarchy process, fuzzy set theory and neural network. Journal of Computational Science, volumen 6, 2015: 23-33.

Lindley D.V. Understanding Uncertainty, Hoboken N.J., John Wiley and Sons, 2006, pp. 226-236.

Maggioni F., Perboli G., Tadei R. The Multi-path Traveling Salesman Problem with Stochastic Travel Costs: Building Realistic Instances for City Logistics Applications Transportation Research. Procedia, volumen 3, 2014: 528-536. 
Marinakis Y., y Marinaki M. A hybrid multi-swarm particle swarm optimization algorithm for the probabilistic traveling salesman problem. Computers And Operations Research, volumen 37 (número 3), 2010: 432-442.

Miragliotta G., Brun A., Soydan I.A. Coordinating multi-business sales through management simulators. International Journal of Production Economics, volumen 121 (número 2), 2009: 533-549.

Morgan M.G. y Henrion M. Uncertainty: A guide to dealing with uncertainty in Quantitative risk and policy analysis, New York, Cambridge University Press, 2004, pp. 102-136.

Paquete L. y Stützle T. Design and analysis of stochastic local search for the multiobjective traveling salesman problem. Computers and Operations Research, volumen 36 (número 9), 2009: 2619-2631.

Rapp A. Outsourcing the sales process: Hiring a mercenary sales force. Industrial Marketing Management, volumen 38 (número 4), 2009: 411-418.

Resnik M.D. Choices, Minneapolis, University Of Minnesota Press, 1987, pp.88-96.

Taha H.A. Operations Research, $9^{\text {na }}$ ed., New Jersey, Prentice Hall, 2011, pp. 395-423.

Tang H. y Miller-Hooks E. Solving a generalized traveling salesperson problem with stochastic customers. Computers and Operations Research, volumen 34 (número 7), 2007: 1963-1987.

Wang Z., Guo J., Zheng M., Wang Y. Uncertain multiobjective traveling salesman problem. European Journal of Operational Research, volumen 241 (número 2), 2015a: 478-489.

Wang P., Sanin C., Szczerbicki E. Evolutionary algorithm and decisional DNA for multiple travelling salesman problem. Neurocomputing, volumen 150 (número A), 2015b: 50-57.

Weyland D., Montemanni R., Gambardella L.M. A metaheuristic framework for stochastic combinatorial optimization pro- blems based on GPGPU with a case study on the probabilistic traveling salesman problem with deadlines. Journal of Parallel and Distributive Computing, volumen 73 (número 1), 2013: 7485.

\section{Citación sugerida:}

\section{Citación estilo Chicago}

Chew-Hernández, Mario Luis, Leopoldo Viveros-Rosas, Verónica Velázquez-Romero. Metodología basada en análisis de Decisiones para distribuir geográficamente una fuerza de ventas. Ingeniería Investigación y Tecnología, XIX, 03 (2018): 255-266.

\section{Citación estilo ISO 690}

Chew-Hernández M.L., Viveros-Rosas L., Velázquez-Romero V. Metodología basada en análisis de decisiones para distribuir geográficamente una fuerza de ventas. Ingeniería Investigación y Tecnología, volumen XIX (número 3), julio-septiembre 2018: 255-266.

\section{SemblanZas de los autores}

Chew-Hernández Mario Luis. Obtuvo el título como ingeniero químico por la UNAM y los grados de maestría en ciencias y doctorado, con especialidad en simulación, por la Universidad de Nottingham, Inglaterra. Desde 2007 es investigador de tiempo completo en el Tecnológico de Estudios Superiores de Coacalco, dentro del área de análisis de decisiones.

Viveros-Rosas Leopoldo. Es ingeniero industrial del Instituto Tecnológico de Tlalnepantla, maestro en calidad por la Universidad Abierta de Tlaxcala y maestro en ciencias en ingeniería industrial por el Tecnológico de Estudios Superiores de Coacalco. Actualmente, es profesor de tiempo completo en esta institución, asignado al área de análisis de decisiones.

Velázquez-Romero Verónica. Es ingeniera industrial de UPIICSA (IPN), maestra en calidad por la Universidad Abierta de Tlaxcala y maestra en ciencias en ingeniería industrial por el Tecnológico de Estudios Superiores de Coacalco. En la actualidad, es investigadora de tiempo completo en esta institución, dentro del área de logística y cadena de suministro. 